\title{
Thyroid nodule: first manifestation of chronic lymphocytic leukaemia
}

\author{
Ana Gonçalves', Sara Vale², Ema Nobre', Ana Paula Barbosa', \\ Ema Piloto', Ana Wessling', Mário Mascarenhas'
}

Serviço de Endocrinologia, Diabetes e Metabolismo do Hospital de Santa Maria, Centro Hospitalar Lisboa Norte ${ }^{2}$ Serviço de Hematologia do Hospital de Santa Maria, Centro Hospitalar Lisboa Norte

\author{
Correspondence to: \\ Ana Pires Gonçalves \\ Rua Sousa Martins, $161 \mathrm{C}$ \\ 218-1050 - Lisboa, Portugal \\ aa.pgoncalves.hsm@gmail.com \\ Received on Feb/17/2014 \\ Accepted on June/11/2014 \\ DOI: 10.1590/2359-3997000000034
}

\begin{abstract}
SUMMARY
The presence of chronic lymphocytic leukaemia (CLL) cells in the thyroid gland is most likely due to a secondary involvement by a systemic disease. The reported incidence of CLL involving the thyroid is extremely low, representing about 3-4\% of all thyroid lymphoproliferative neoplasm. We report a rare case of CLL presenting initially in the thyroid gland. Systemic disease was detected as a result of thyroid investigation. An 85 years old woman, with multinodular goiter without adenophaties, was referred to our department, carrying a fine needle aspiration biopsy (FNAB) report of a private institution referring "lymphoid monomorphic proliferation" and suggesting a "Core-needle biopsy" for further investigation. She was euthyroid (TSH- $0.5 \mathrm{uU} / \mathrm{mL}$ (0.4-4.0), thyroid antibodies negative, including TRab). The patient denied systemic symptoms and at physical examination there were no adenophaties or organomegalies. FNAB analysis was repeated. Although the patient denied constitutional symptoms and there were no relevant findings in physical examination, technetium $99 \mathrm{~m}$ thyroid gamagraphy (GG) and blood count were additionally asked. FNAB analysis concluded lymphocytic tiroiditis, but thyroid GG revelled global hypocaptation and blood count showed $173.4 \times 10^{9}$ leukocyte/L with $94 \%$ lymphocyte. An ecoguided FNAB with flow cytometry identified thyroid infiltration by monotonous population of blasts with phenotype consistent with $\mathrm{CLL} /$ malignancy of mature B-cells. CLL/malignancy of mature B-cells was also detected in peripheral blood analysis, suggesting systemic disease with secondary thyroid involvement. The patient started chemotherapy with rituximab and chlorambucil with good response. Pos-treatment GG revelled "Increased levels of uptake in the middle third of the right lower lobe, with low uptake of the remaining parenchyma". In conclusion, good communication with the pathologist can improve diagnostic accuracy and dictate appropriate therapy. The use of techniques such as flow cytometry, immunoglobulin gene rearrangements, and immunohistochemistry has improved diagnostic accuracy and obviated more invasive procedures, such as core needle or open surgery biopsy. Apart from chemotherapy, immunochemotherapy with anti-CD20 and anti-CD52 monoclonal antibodies can be used in the treatment of CLL. Arch Endocrinol Metab. 2015;59(2):190-4
\end{abstract}

\section{INTRODUCTION}

$\mathrm{C}$ hronic lymphocytic leukaemia (CLL) is a lowgrade indolent neoplasm composed of small B lymphocytes in the peripheral blood, bone marrow, lymph nodes, and other tissues $(1,2)$. The diagnosis of CLL requires the presence of more than $5 \times 10^{9}$ small monoclonal lymphocytes per liter in the peripheral blood (3). Flow cytometry will demonstrate clonality, as determined by kappa or lambda light chain restriction, and CLL cells are typically positive for CD5, CD23, CD19, and CD20 (3).

The presence of CLL cells in the thyroid gland is most likely due to a secondary involvement by a systemic disease $(2,4)$.

The reported incidence of CLL involving the thyroid is extremely low, representing about 3\% to $4 \%$ of all thyroid lymphoproliferative neoplasm (5-8).
Similarly, primary thyroid lymphoma is a rare disease. It is defined as a lymphomatous process involving the thyroid gland without continuous spread or other areas of involvement. The most common presentation is a rapidly enlarging thyroid mass that can lead to compressive symptoms.

We report a rare case of CLL presenting itself initially in the thyroid gland. The presence of systemic disease was detected as a result of thyroid investigation.

\section{CASE REPORT}

An 85 years old woman, with multinodular goiter without adenophaties, was referred to our department, carrying a fine needle aspiration biopsy (FNAB) report of a private institution referring "lymphoid monomorphic proliferation" and suggesting a "Core-needle biopsy" for further investigation. 
She was euthyroid (TSH-0.5 uU/mL [0.4-4.0], thyroid antibodies negative, including TRab). The patient denied systemic symptoms and at physical examination there were no adenophaties or organomegalies.

The case was discussed with the pathological anatomy department. Since the slides from the previous FNAB were unavailable, the procedure was repeated. Although the patient denied constitutional symptoms and there were no relevant findings in physical examination, blood count and technetium $99 \mathrm{~m}$ thyroid gamagraphy (GG) were additionally asked.

FNAB analysis concluded lymphocytic tiroiditis, but thyroid GG revelled global hypocaptation (Figure 1) and blood count showed $173.4 \times 10^{9}$ leukocyte/L with $94 \%$ lymphocyte. The patient was referred to haematology department. Considering gamagraphyc results and negative thyroid autoimmune study, an ecoguided FNAB with flow cytometry was ordered. Thyroid infiltration by monotonous population of blasts was identified with phenotype consistent with LLC/malignancy of mature B-cells (positivity for CD19 (98\%), CD5, 20, 22, 23, 45, lambda chains) (Figures 2 and 3). Peripheral blood analysis identified a monoclonal B lymphoid population. The immunophenotype was identical to the one found in thyroid aspirate, suggesting secondary involvement by a systemic disease (Figure 4). Computed tomography (Tc) of the chest, abdomen, and pelvis did not reveal any evidence of other involvement (Figure 4). The diagnosis was established on basis of peripheral blood count and flow cytometry. Myelogram was not performed considering patient's advanced age and it's little diagnostic value in this context.

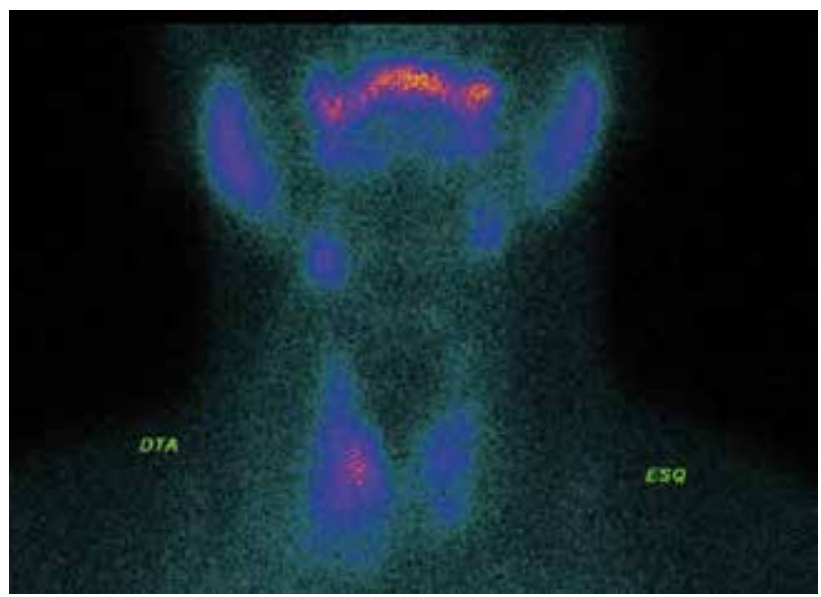

Figure 1. Technetium $99 \mathrm{~m}$ thyroid gamagraphy: thyroid with global hypocaptation.

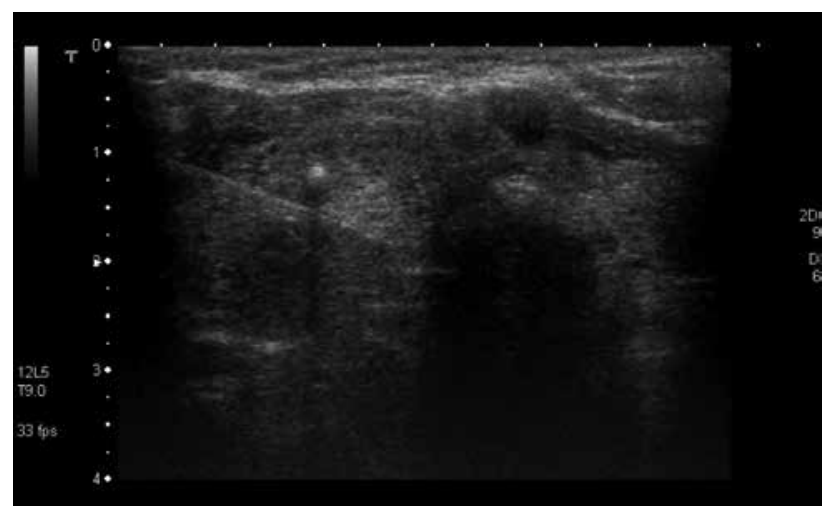

Figure 2. Ecoguided FNAB was made to dominant nodule in the right lobe of the thyroid gland: $24 \times 22 \mathrm{~mm}$, solid, hyperecogenic.
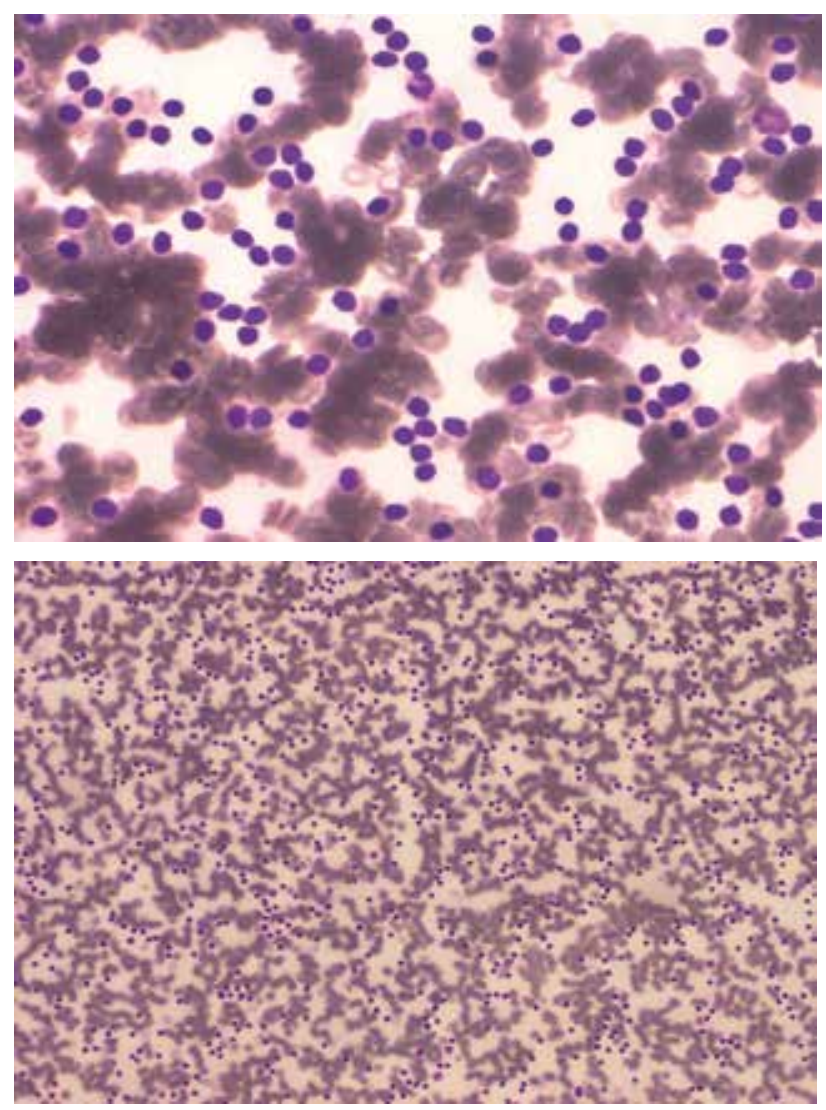

Figure 3. Anatomopathological exam of thyroid cytology revelling thyroid infiltration by monotonous population of blasts.
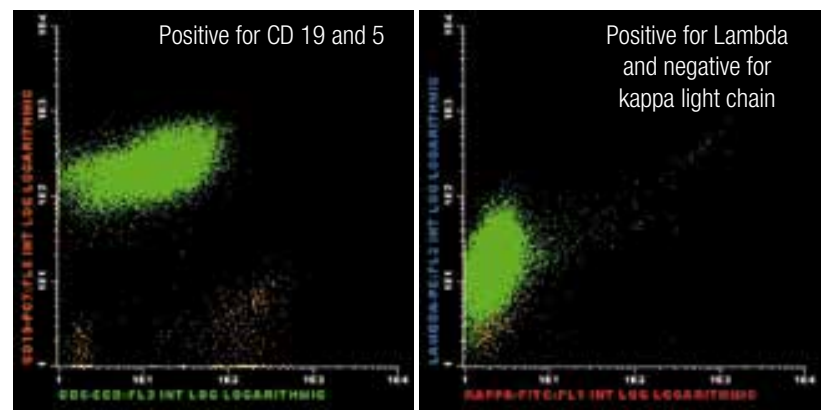

Figure 4. Flow cytometry of thyroid aspirated cytology. 
The patient was classified as stage 0 de Rai-Sawitsky system and stage A de Binet classification, corresponding to good prognostic disease. The patient was treated with rituximab and chlorambucil during 6 months with good response. On last evaluation, hemogram revealed 4,000 x $10^{6}$ leukocytes/L, and $18.5 \%$ lymphocytes. Tc showed "thyroid with marked heterogeneity, outlining multiple hypodense nodular areas. No evidence of lymphadenopathy or other suspicious images" (Figure 5).
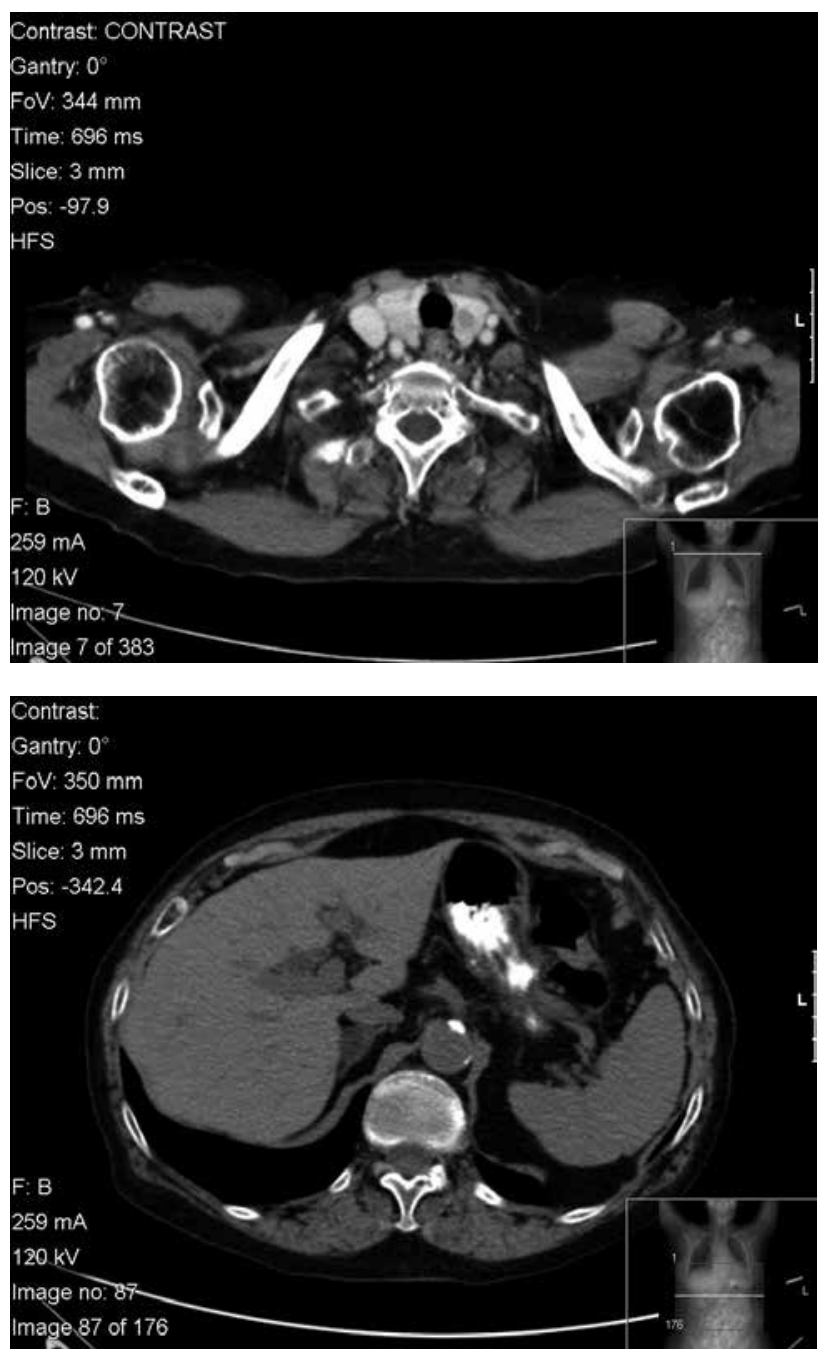

Figure 5. Staging: computed tomography of cervical region, chest, abdomen, and pelvis without other organomegalies.

Simultaneously, thyroid function was monitored, remaining stable. A GG was ordered and revelled "increased levels of uptake in the middle third of the right lower lobe, with low uptake of the remaining parenchyma" (Figure 6).
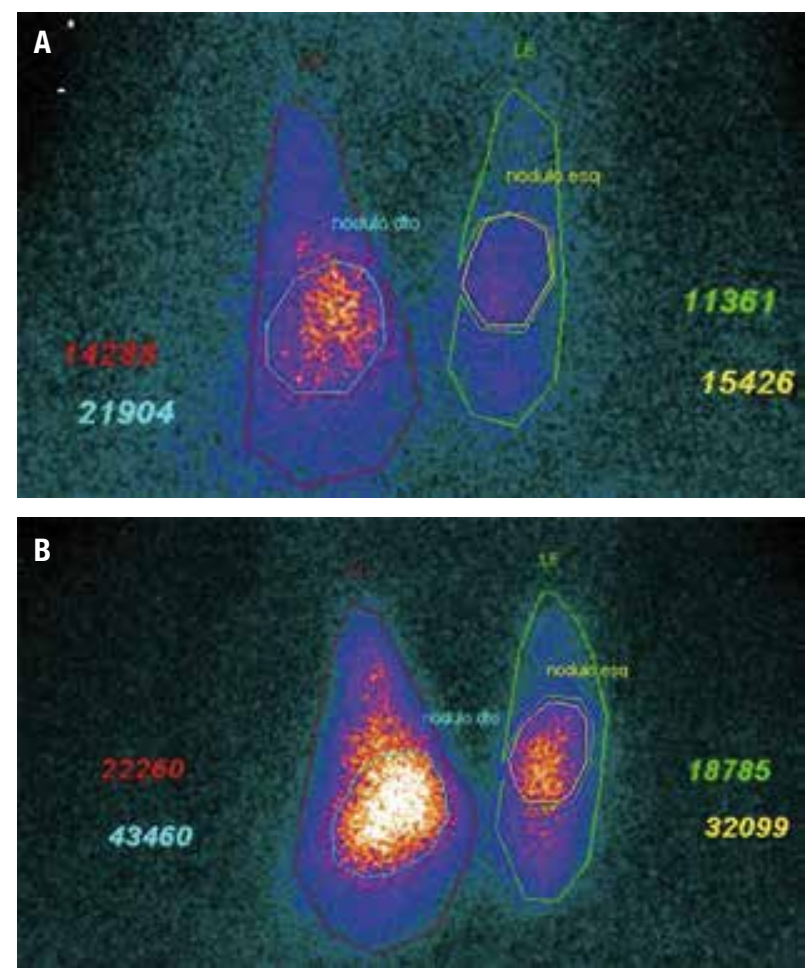

Figure 6. Technetium 99m thyroid gamagraphy - comparative images: (A) Before treatment; (B) After treatment: "Increased levels of uptake in the middle third of the right lower lobe, with low uptake of the remaining parenchyma" 3 months after treatment.

\section{DISCUSSION}

Lymphoproliferative neoplasias account for approximately $2 \%$ of all malignancies of the thyroid gland $(9,10)$. The most frequent histologic types are primary thyroid lymphomas, particularly non-Hodgkin's type with a B-cell phenotype. Diffuse large B-cell lymphoma (DLCL) accounts for about $50 \%$ of all cases and MALT lymphoma (mucosa associated lymphoid tissue) represents approximately $23 \%$ of all cases $(10,11)$.

Eighty percent of primary thyroid lymphoma arises in a background of chronic lymphocytic thyroiditis, which poses diagnostic difficulties. Likewise, cytological diagnosis of MALT lymphomas is very difficult because they are composed of a heterogeneous infiltrate composed of a spectrum of small and large lymphoid cells.

The reported incidence of CLL involving the thyroid is extremely low, representing about $3-4 \%$ of all thyroid lymphoproliferative neoplasias (5-8). The presence of CLL cells in the thyroid gland is most likely due to a secondary involvement by a systemic disease $(2,4)$. 
Pathological proliferation of B-cells occurs in patients with CLL. These cells accumulate as a result of impaired apoptosis, infiltrating bone marrow, lymph nodes, spleen and, in very rare cases, other organs $([12,13)$. This type of leukaemia develops in patients between 65 and 70 years of age, more frequently in males, and it may remain asymptomatic for extended time. General symptoms, such as excessive sweating, weight loss, fever, and recurrent infections, including opportunistic infections, may occur, as well as lymphadenopathy and enlargement of the spleen and the liver. Peripheral blood cell count is characterized by lymphocytosis, thrombocytopenia and anaemia. Therapy depends on clinical stage of the disease and the presence of general symptoms. Apart from chemotherapy, immunochemotherapy with anti-CD20 and anti-CD52 monoclonal antibodies is used in the treatment of CLL (14).

The sonographic appearance of thyroid lymphoma has most commonly been described as a pseudocystic or hypoechoic mass; however, these characteristics are not universal (15). Most patients are eutiroid and present one or more cold nodules on thyroid scan. Fortunately for our patient, the presence of a well-defined thyroid nodule, initiated the workup.

In this case, although the first cytology in our institution was consistent with autoimmune thyroiditis, the patient had negative thyroid anti-antibodies and global hypocaptation at the gamagraphic study, which led to the performance of an FNAB with flow cytometry and enabled the diagnosis.

Thyroid lymphoma can be very difficult to distinguish histologically from Hashimoto's thyroiditis on FNAB $(5,7,8)$. The use of techniques such as flow cytometry, immunoglobulin gene rearrangements, and immunohistochemistry has improved diagnostic accuracy and obviated more invasive procedures, in this case, core needle biopsy $(8,16)$.

The technique of FNAC is a valuable method for investigation of thyroid tumours. However cytological diagnosis of lymphoid lesions, particularly primary thyroid lymphomas, is very challenging. The principal difficulty is the differentiation of low-grade lymphoma of thyroid from autoimmune thyroiditis. Even, conjugation of morphologic criteria with immunocytochemical staining yields $30 \%$ of false negative results. False negative results seem to be associated to sampling error, because low-grade B cell MALT lymphoma originates and coexist with chronic autoimmune tiroiditis. Adequate sampling by means of several needle punc- tures ecoguided and always keep in mind the possibility of lymphoma when smears exhibit typical features of chronic autoimmune tiroiditis are key to improve diagnosis.

Our patient was asymptomatic and presented thyroid infiltration, without lymphadenopathy or hepatosplenomegaly, and high-grade leukocytosis and anaemia, without thrombocytopenia. In this case, thyroidal involvement justified treatment initiation.

In conclusion, thyroid involvement of lymphoproliferative neoplasm is a rare disease that can pose diagnostic difficulty for clinicians. Thyroid lymphoproliferative neoplasms can be very difficult to distinguish histologically from Hashimoto's thyroiditis on FNAB. Consequently the diagnosis is often delayed or failed, involving inappropriate treatment and morbidity for the patients. Good communication with the pathologist can enhance diagnostic accuracy and dictate appropriate therapy.

We presented a clinical case of one of the most uncommon subtypes of lymphoproliferative neoplasia involving the thyroid. In this patient, CLL was identified initially in the thyroid gland, and the presence of systemic involvement was established afterwards. The use of flow cytometry has enabled the diagnosis and obviated more invasive procedures, as core needle biopsy. Interestingly, the patient was asymptomatic and presented thyroid infiltration without other organ involvement. The patient was classified as low risk and thyroid involvement was decisive for treatment initiation.

This clinical case also highlights that chemotherapy associated to immunochemotherapy with anti-CD20 and anti-CD52 is effective for CLL treatment.

Fundings: nothing to declare.

Disclosure: no potential conflict of interest relevant to this article was reported.

\section{REFERENCES}

1. Derringer GA, Thompson LD, Frommelt RA, Bijwaard KE, Heffess CS, Abbondanzo SL. Malignant lymphoma of the thyroid gland. Am J Surg Pathol. 2000;24:623-39.

2. Kossev P, Livolsi V. Lymphoid lesions of the thyroid: review in light of the revised European-American Iymphoma classification and upcoming World Health Organization classification. Thyroid. 1999;9:1273-80.

3. Kaufman M, Rubin J, Rai K. Diagnosing and treating chronic lymphocytic leukemia in 2009. Oncology. 2009;23:1030-7.

4. Reid-Nicholson M, Moreira A, Ramalingam P. Cytologic features of mixed papillary carcinoma and chronic lymphocytic leukemia/ 
small lymphocytic lymphoma of the thyroid gland. Diagn Cytopathol. 2008;36:813-7.

5. Widder S, Pasieka JL. Primary thyroid lymphomas. CurrTreat Options Oncol. 2004;5:307-13.

6. Graff-Baker A, Roman SA, Thomas DC, Udelsman R, Sosa JA. Prognosis of primary thyroid lymphoma: demographics, clinical, and pathologic predictors of survival in 1,408 cases. Surgery. 2009;146:1105-15.

7. Thieblemont $C$, Mayer A, Dumontet C, Barbier Y, Callet- Bauchu $\mathrm{E}$, Felman $\mathrm{P}$, et al. Primary thyroid lymphoma is a heterogeneous disease. J Clin Endocrinol Metab. 2002;87:105-11.

8. Cha $\mathrm{C}$, Chen H, Westra WH, Udelsman R. Primary thyroid lymphoma: can the diagnosis be made solely by fine-needle aspiration? Ann Surg Oncol. 2002;9:298-302.

9. Sangalli G, Serio G, Zampatti C, Lomuscio G, Colombo L. Fine needle aspiration cytology of primary lymphoma of the thyroid: a report of 17 cases. Cytopathology. 2001;12:257-63.

10. Thieblemont $C$, Mayer A, Dumontet $C$, Barbier $Y$, Callet-Bauchu $\mathrm{E}$, Felman $\mathrm{P}$, et al. Primary thyroid lymphoma is a heterogeneous disease. J Clin Endocrinol Metab. 2002;87:105-11.
11. Harris NL, Jaffe ES, Diebold J, Flandrin G, Muller-Hermelink HK, Vardiman J, et al. The World Health Organization classification of neoplastic diseases of the hematopoietic and lymphoid tissues. Report of the Clinical Advisory Committee meeting, Airlie House, Virginia, November, 1997. Ann Oncol. 1999;10:1419-32.

12. Derringer GA, Thompson LD, Frommelt RA, Bijwaard KE, Heffess CS, Abbondanzo SL. Malignant lymphoma of the thyroid gland: a clinicopathologic study of 108 cases. Am J Surg Pathol. 2000;24:623-39.

13. Wirtzfeld DA, Winston JS, Hicks WL Jr, Loree TR. Clinical presentation and treatment of non-Hodgkin's lymphoma of the thyroid gland. Ann Surg Oncol. 2001;8:338-4.

14. Graff-Baker A, Roman SA, Thomas DC, Udelsman R, Sosa JA. Prognosis of primary thyroid lymphoma: demographic, clinical, and pathologic predictors of survival in 1,408 cases. Surgery. 2009;146:1105-15.

15. Foppiani L, Secondo V, Arlandini A, Quilici P, Cabria M, Del Monte P. Thyroid lymphoma: a rare tumor requiring combined management. Hormones. 2009;8:214-8.

16. Pasieka JL. Hashimoto's disease and thyroid lymphoma: role of the surgeon. World J Surg. 2000;24:966-70. 